\title{
Responses to song playback differ in sleeping versus anesthetized songbirds
}

\author{
Sarah W. Bottjer ${ }^{1}$, Chloé ${ }^{2}$ Le Moing, Ellysia Li, Rachel Yuan ${ }^{3}$
}

Section of Neurobiology, University of Southern California, Los Angeles, CA 90089 USA

${ }^{1}$ correspondence bottjer@usc.edu

${ }^{2}$ current affiliation: Oregon Health \& Science University

${ }^{3}$ current affiliation: University of Oregon, Knight Campus 


\begin{abstract}
Vocal learning in songbirds is mediated by a highly localized system of interconnected forebrain regions, including recurrent loops that traverse the cortex, basal ganglia, and thalamus. This brain-behavior system provides a powerful model for elucidating mechanisms of vocal learning, with implications for learning speech in human infants, as well as for advancing our understanding of skill learning in general. A long history of experiments in this area has tested neural responses to playback of different song stimuli in anesthetized birds at different stages of vocal development. These studies have demonstrated selectivity for different song types that provide neural signatures of learning. In contrast to the ease of obtaining responses to song playback in anesthetized birds, song-evoked responses in awake birds are greatly reduced or absent, indicating that behavioral state is an important determinant of neural responsivity. Song-evoked responses can be elicited in sleeping as well as anesthetized zebra finches, and the selectivity of responses to song playback in adult birds tends to be highly similar between anesthetized and sleeping states, encouraging the idea that anesthesia and sleep are highly similar. In contrast to that idea, we report evidence that cortical responses to song playback in juvenile zebra finches (Taeniopygia guttata) differ greatly between sleep and urethane anesthesia. This finding indicates that behavioral states differ in sleep versus anesthesia and raises questions about relationships between developmental changes in sleep activity, selectivity for different song types, and the neural substrate for vocal learning.
\end{abstract}

\title{
Significance
}

Patterns of spiking activity based on electrophysiological recordings in many different taxa are known to be heavily dependent on behavioral state. Neural activity patterns are frequently similar between sleep and anesthesia, which has encouraged the idea that similar states characterize sleep and anesthesia. Based on comparisons across studies, we report that activity patterns are highly dissimilar between sleep and urethane anesthesia in a cortical region of juvenile songbirds. These data argue against the idea that similar behavioral states are achieved in sleep versus anesthesia. 


\section{Introduction}

Vocal learning in zebra finches serves as a powerful model for investigating mechanisms of motor skill learning during development (Doupe and Kuhl, 1999; Brainard and Doupe, 2013). Juvenile zebra finches learn the sounds used for vocal communication, and this type of skill learning, like other forms of goaldirected learning, is controlled by cortico-basal ganglia circuits (Yin and Knowlton, 2006; Graybiel, 2008; Redgrave et al., 2010; Turner and Desmurget, 2010; Cox and Witten, 2019). Similar to infants learning speech, juvenile songbirds memorize the vocal sounds of their adult tutor. They then progressively refine their own vocal behavior to imitate the tutor song (the goal behavior) during the sensorimotor stage of vocal learning. This process requires the evaluation of feedback of self-generated vocalizations against a neural representation of the goal tutor song to guide the gradual acquisition of an accurate imitation.

Neural control of vocal learning in juvenile zebra finches is vested in basal ganglia loops that emanate from the cortical nucleus LMAN (Fig. 1) (Bottjer et al., 1984; Scharff and Nottebohm, 1991; Aronov et al., 2008). CORE and SHELL subregions of LMAN make parallel connections through the basal ganglia and thalamus (Johnson et al., 1995; lyengar et al., 1999; Luo et al., 2001; lyengar and Bottjer, 2002b; Bottjer, 2004; Gale et al., 2008; Person et al., 2008; Paterson and Bottjer, 2017). The CORE pathway mediates vocal motor production in juvenile songbirds (Bottjer et al., 1984; Scharff and Nottebohm, 1991; Aronov et al., 2008; Elliott et al., 2014; Kojima et al., 2018) and is functionally similar to sensorimotor corticobasal ganglia loops in mammals that contribute to learning and performance (Alexander and Crutcher, 1990; Graybiel, 2008; Yin et al., 2009; Ashby et al., 2010; Redgrave et al., 2010; Thorn et al., 2010; Gremel and Costa, 2013; Kupferschmidt et al., 2017). In contrast, the SHELL pathway is involved in evaluating sensorimotor performance and is functionally similar to associative-limbic loops that traverse the basal ganglia; lesions in the SHELL pathway of juvenile birds impair the ability to imitate tutor song, but do not cause motor disruption of song production (Bottjer and Altenau, 2010). This disruption of learning but not motor performance suggests that SHELL circuitry may help to evaluate whether selfgenerated vocalizations match learned tutor sounds.

Studies of the mechanisms that underlie vocal learning in songbirds have a long and venerable history of examining neural responses to playback of different song types in anesthetized juvenile and adult birds (Margoliash, 1983, 1986; Volman, 1993; Lewicki and Konishi, 1995; Solis and Doupe, 1997, 1999, 2000; Adret et al., 2012). One quest in this area was to discover a population of neurons that encode the tutor song memorized by each juvenile bird. Achiro and Bottjer (2013) reported that the SHELL subregion of LMAN in juvenile anesthetized birds contains a large proportion of neurons ( $30 \%)$ that respond significantly only to playback of tutor song. This tutor-tuned population provides a target memory that is essential for matching self-generated utterances to the goal tutor song, and is present only during early stages of sensorimotor integration. The proportion of tutor-tuned neurons diminishes during development as the incidence of neurons that responds selectively to each bird's own song increases, suggesting that tutor-tuned neurons are lost (Johnson and Bottjer, 1992, 1993, 1994) or re-tuned to provide a template of self-generated song (Volman, 1993; Zevin et al., 2004; Nick and Konishi, 2005a; Kojima and Doupe, 2007; Achiro and Bottjer, 2013). In accord with the latter idea, the emergence of 
selectivity for each bird's own song is a ubiquitous signature of vocal learning across forebrain regions including HVC, LMAN, RA, basal ganglia, and thalamus (Margoliash, 1983; Margoliash and Konishi, 1985; Margoliash, 1986; Margoliash and Fortune, 1992; Volman, 1993; Doupe, 1997; Solis and Doupe, 1997; Person and Perkel, 2007).

Data based on playback of songs in anesthetized birds has highlighted the power of such experiments for studying mechanisms of vocal learning. However, several studies have shown that behavioral state is an important determinant of neural responsivity to song playback. Song-evoked responses can be elicited in sleeping as well as anesthetized zebra finches, and responses to song playback in adult birds tend to be highly similar between anesthetized and sleeping states (Dave et al., 1998; Dave and Margoliash, 2000; Nick and Konishi, 2001), encouraging the idea that anesthesia and sleep states are highly similar. In contrast, song-evoked responses are greatly diminished or absent in awake zebra finches (Schmidt and Konishi, 1998; Cardin and Schmidt, 2003; Rauske et al., 2003; Cardin and Schmidt, 2004b, a), which is reminiscent of the suppression of auditory responses to self-generated sounds in both vertebrate and invertebrate taxa (Suga and Shimozawa, 1974; Poulet and Hedwig, 2006, 2007; Eliades and Wang, 2008; Singla et al., 2017) (see Discussion). Here we report that responses to playback of different song types in both CORE and SHELL subregions of LMAN in sleeping juvenile birds are substantially different from those reported previously in urethane-anesthetized birds of the same age (Achiro and Bottjer, 2013). This difference is consistent with recent data showing that urethane anesthesia does not mimic sleep states (Mondino et al., 2021).

\section{Materials and Methods}

Subjects. All procedures were performed in accordance with the [Author University] animal care committee's regulations. Five juvenile male zebra finches (Taeniopygia guttata) were used. Birds were bred in group aviaries and remained with their natural parents up until 33 days post-hatch (dph), at which time they and their father were removed from the main aviary and housed in an individual cage in the recording chamber in order to habituate them to the space. Experimental birds therefore received normal social-auditory experience and exposure to the tutor song (their father's song) (Böhner, 1983, 1990; Mann et al., 1991; Mann and Slater, 1995; Roper and Zann, 2006).

Electrophysiology. At $39 \mathrm{dph}$ birds were anesthetized with isoflurane (1.5-1.8\% inhalation) and placed in a stereotaxic apparatus. An electrode assembly consisting of eight tungsten-wire stereotrodes affixed to a movable microdrive was attached to the skull using dental cement such that the stereotrodes were implanted $\sim 300 \mathrm{~mm}$ dorsal to LMAN CORE and SHELL. Each stereotrode was a twisted pair of polyester polyamide-imide overcoated tungsten wires (25 um diameter, California Fine Wire company, Grover Beach, CA) routed through fused silica capillary tubing (200 $\mu \mathrm{m}$ diameter). The assembly consisted of four posterior stereotrodes and four anterior stereotrodes; a silver wire, placed between the skull and skin, served as animal ground. Following surgery each bird was housed in a small individual cage in the recording chamber adjacent to the cage with the father; the father was removed 4-6 days later. 
One to two days following surgery, the stereotrode assembly was connected to a recording headstage (HS-16, Neuralynx, Bozeman, MT) with a flexible cable connected to a commutator (PSR, Neuralynx); 15 channels of neural data were amplified, band passed between 300 and $5000 \mathrm{~Hz}$ (two Lynx-8 amplifiers, Neuralynx), and digitized at $32 \mathrm{kHz}$ using Spike2 software (Power 1401 data acquisition interface, Cambridge Electronic Design). Audio and video were recorded coincident with neural activity: vocalizations were recorded to the $16^{\text {th }}$ channel using a lavalier microphone (Sanken COS-11D) mounted in the cage, and two USB-video cameras (30 FPS, ELP Day Night Vision, X000UPN1M5, HD 1080p) were placed at the front and side of the cage to record video files aligned to the neural activity. Two consecutive 60-min recordings were made between approximately 8 and 10 P.M. starting about one hour after lights off. Stereotrodes were manually advanced with the microdrive on consecutive days in the afternoon. The range of ages when recordings were made from LMAN CORE and/or SHELL ranged from 43 to 53 with a mean of $48.5 \mathrm{dph}$.

All birds received playback of four different songs: the bird's own song (OWN, recorded within 24 hours prior to each recording), the bird's tutor song (TUT), a juvenile conspecific song (JuvCon), and an adult conspecific song (AdICon). The latter two songs served as control stimuli for OWN song and TUT song, respectively. JuvCon songs were age-matched to the age of the experimental bird's OWN songs. The order of stimuli within a block of four songs was random without replacement, and the inter-stimulus interval was $30 \pm 1 \mathrm{sec}$. Each song type was played back approximately 50 times at an amplitude of 56$59 \mathrm{~dB}$, but only playbacks that occurred during sleeping periods were used for analysis (see below).

At the end of each experiment, birds were perfused ( $0.7 \%$ saline followed by $10 \%$ formalin), and brains were removed and postfixed before being cryo-protected ( $30 \%$ sucrose solution) and frozen-sectioned in the coronal plane (50 $\mu \mathrm{m}$ thick). Sections were Nissl stained with thionin to visualize stereotrode tracks and verify recording locations. The border between CORE and SHELL subregions of LMAN can be distinguished based on the density of magnocellular somata, which is low in SHELL relative to CORE.

Data Analysis. A recording site was considered for analysis if it was confirmed histologically to be in either LMAN-CORE or LMAN-SHELL (excluding $50 \mu \mathrm{m}$ on either side of the CORE/SHELL border). The evoked responses of LMAN neurons tend not to persist throughout song stimuli longer than $1 \mathrm{sec}$, as reported previously (Doupe, 1997; Solis and Doupe, 1997, 1999; Kojima and Doupe, 2007; Achiro and Bottjer, 2013). Therefore, response strengths calculated for song stimuli longer than $1 \mathrm{sec}$ underestimate the actual response by averaging across both the early phasic response and the period of decreased response. To correct for this stimulus duration bias (e.g., longer songs underestimate true response strengths), all analyses were performed using neural data collected during the first second of song playback.

Periods of sleep were scored manually by two independent observers; as a conservative estimate, only periods ranked as sleep by both observers were used for analysis. Careful examination of the video files was used to mark sleeping periods as those in which birds were completely quiescent, displaying a regular pattern of deep rhythmic breathing with their eyes closed for at least 10 seconds. Sleeping periods were terminated at least 2 seconds prior to onset of large movements (e.g., wing movements) 
or eye-opening. As indicated above, only song playbacks that occurred during sleeping periods were included for analysis; the number of playbacks ranged from 16 to 48 (average 33 playbacks per song type in SHELL and 28 in CORE).

Movement artifact in multiunit neural recordings was correlated across recording channels and was eliminated or reduced using offline common average referencing: for each recording channel, the signal across the 14 remaining recording channels was averaged and subtracted from that channel to remove movement artifact (Ludwig et al., 2009). Noise was calculated as the standard deviation of the entire (2hour) voltage recording, and minimum signal-to-noise ratio was set as three times the standard deviation; this threshold was used for spike detection. Single units were sorted from multiunit data by first automatically clustering units with KlustaKwik (KD Harris, University College London). KlustaKwik clusters were manually inspected across 18 different waveform features and further refined using MClust 4.4 (A. D. Redish, University of Minnesota). Clusters were included for analysis if $<1 \%$ of spikes had an interspike interval $(I S I)<2$ ms.

We determined whether each single unit was responsive to song playback by testing for a significant change in firing rate (excitation or suppression) between baseline and each song type (Wilcoxon signedrank test, $p<0.05$ ). Baseline periods were defined as 1 -sec periods immediately prior to stimulus playback, with the restriction that they must fall within sleep periods. For each song playback, the firing rates during the two closest baseline periods were averaged to generate a corresponding baseline value. To compare differences in firing rates across neurons, standardized response strengths (RS) were calculated as:

$$
\text { response strength }(\mathrm{RS})=\frac{\bar{S}-\bar{B}}{\sqrt{\operatorname{Var}(S)+\operatorname{Var}(B)-2 * \operatorname{Covar}(S, B)}}
$$

Where $S$ is the average firing rate (spikes/sec) during stimulus, and $B$ is the average firing rate during baseline, such that a positive value indicates an increased rate to a stimulus (excitation) and a negative value indicates a decreased rate (suppression).

To measure song selectivity for each song type for each cell, a difference score was calculated for "Song $A^{\prime \prime}$ as follows: Song $A_{\triangle R S}=R S_{\text {songA }}-R_{\text {songB }}$. For example, positive scores obtained by subtracting response strengths to OWN, AdICon, and TUT (comparison songs) from JuvCon (reference song) would indicate selectivity for JuvCon. This measure is similar to the psychometric discriminability index d' except that responses are standardized before being subtracted, as opposed to subtracting response strengths and then dividing by the standard deviations as in d' (cf. Achiro \& Bottjer 2013). Difference scores for song-suppressed responses were reversed in sign so that a positive difference score indicates a preference for the reference song over the comparison song while a negative difference score indicates a preference for the comparison song (see Results).

Statistics. We used non-parametric statistics due to non-normal distributions of data, differing numbers of responses between song types, and differing numbers of neurons between CORE and SHELL regions. Differences in proportions were tested using $\chi^{2}$ tests, and differences in distributions were tested with 
Kolmogorov-Smirnov $Z$ tests. Friedman tests were used to evaluate differences in RS between song types (as a repeated measure) within CORE and SHELL regions, whereas Kruskal-Wallis tests were used to evaluate differences between CORE and SHELL. Wilcoxon signed-rank tests were used to assess individual differences between song types, including Benjamini-Hochberg corrections for multiple comparisons (Benjamini and Hochberg, 1995). All values given as mean \pm SEM.

\section{Results}

Different song types elicited different proportions of responses in both CORE and SHELL regions of LMAN

We recorded from CORE and SHELL subregions of LMAN in sleeping juvenile zebra finches (43 to $53 \mathrm{dph}$, mean $=48.5 \mathrm{dph}$ ). By this age juveniles have completed memorization of their tutor's song and begun to practice their incipient song vocalizations. All neurons ( $n=66$, CORE; $n=104$, SHELL) were tested with four different song types: each bird's own song (OWN), each bird's tutor song (TUT), an agematched song from a juvenile conspecific (JuvCon) and an adult conspecific (AdICon). Approximately half of the neurons in both CORE and SHELL showed a significant change in firing rate to at least one of the song types presented (CORE: 0.53, 35/66; SHELL: 0.47, 49/104); thus both regions showed similar levels of responsivity to song playback $\left(\chi^{2}=0.57, p=0.45\right)$. Proportions of significant playback responses varied by song type within both CORE and SHELL (CORE: $\chi^{2}=13.8, p=0.003$; SHELL: $\chi^{2}=12.6$, $p=0.006$ ) (Fig. 2 top panel; Table 1 ). JuvCon song elicited the highest proportion of responses whereas TUT evoked the lowest. Individual comparisons showed that the incidence of evoked responses to JuvCon was higher than that to TUT (CORE: $p=0.003$; SHELL: $p=0.01$, Fisher's exact test, BenjaminiHochberg corrected). JuvCon song also elicited a higher proportion of responses in LMAN-CORE neurons compared to AdlCon song ( $p=0.04)$; no other comparisons between JuvCon and other song types were significant ( $p>0.09$ or higher).

Individual neurons were not broadly tuned: almost all neurons responded to either one or two of the four song types played; CORE neurons responded to $1.26 \pm 0.07$ different songs on average, whereas SHELL neurons responded to $1.29 \pm 0.08$. Figure 3 (left) shows that approximately $75 \%$ of neurons in both CORE and SHELL subregions responded to only one song type; the majority of the remaining cells responded to only two song types - no CORE neurons responded to three songs whereas $4 \%$ of SHELL neurons responded to three songs. The right side of Figure 3 depicts the song types to which each neuron responded, confirming that a low proportion of neurons in both CORE and SHELL responded to playback of TUT song, while relatively high proportions responded to both JuvCon and OWN songs.

Although we found evidence of both excitation and suppression, the majority of cells within both CORE and SHELL showed only suppressed responses. Approximately $75 \%$ of cells in CORE and SHELL were suppressed by song playback, whereas relatively few cells responded with only excitation or a combination of excitation and suppression to different song types (Table 2). The dominance of suppressed responses was clear for all four song types, but was particularly pronounced for the two song types that elicited the highest percentage of responses - JuvCon and OWN (Table 1). We therefore examined the proportions of suppressed responses elicited by different song types (Fig. 2B). In contrast 
to comparison across all playback responses (Fig. 2 top), the bottom panel of Figure 2B shows that only SHELL neurons showed differential suppression between song types (SHELL: $\chi^{2}=16.0, p=0.001$; CORE: $\chi^{2}=4.99, p=0.173$ ). Within SHELL neurons, JuvCon evoked a higher incidence of suppressed responses compared to both TUT and AdICon, but not OWN (TUT: $p=0.006$; AdICon: $p=0.034$, Fisher's exact test, Benjamini-Hochberg corrected). OWN song also evoked a higher proportion of suppressed responses compared to TUT (TUT: $p=0.011$; AdICon was marginally significant, $p=0.057$; Fisher's exact test, Benjamini-Hochberg corrected). Thus, the proportion of suppressed responses varied by song type in SHELL, but not CORE; within SHELL neurons both JuvCon and OWN elicited a high incidence of suppressed responses relative to AdICon and (especially) TUT.

We are confident that we measured responses to song playback during periods of sleep since the use of behavioral criteria has been shown to be highly reliable (Szymczak et al., 1996; Low et al., 2008). We also noted that spontaneous firing rates (spikes/sec) during the night were higher during periods marked as waking compared to sleep periods (CORE waking 1.96, sleeping 1.51; SHELL waking 2.51, sleeping 2.07; Wilcoxon signed-ranks tests $p<0.0001$ for both CORE and SHELL). To summarize data based on neuronal proportions in sleeping juvenile birds: 1 ) neurons in both subregions of LMAN responded in a selective fashion to song stimuli in sleeping birds during the period of early sensorimotor learning; 2) all individual songs were more likely to elicit suppression of firing rates rather than excitation, especially for JuvCon and OWN songs; 3) SHELL neurons showed a greater tendency towards suppressed responses to JuvCon and OWN songs compared to CORE neurons. Neurons at the population level evinced a preference for juvenile songs over adult songs, regardless of whether the juvenile song was self-generated (OWN) or produced by an age-matched conspecific bird (JuvCon).

This overall pattern of results contrasts markedly with that observed in a previous study in which birds of the same age were urethane-anesthetized rather than sleeping (Achiro and Bottjer, 2013). In that study, neurons in CORE were more likely to respond to playback compared to those in SHELL (0.89 versus 0.68 ), and neurons in both CORE and SHELL were much more likely to show excitation: CORE neurons never showed suppressed responses whereas $\sim 80 \%$ of responses in SHELL neurons were excitatory and $\sim 20 \%$ were suppressed. In addition, a large proportion of SHELL neurons exhibited a significant response only to TUT compared to those in CORE (0.28 versus 0.04$)$, whereas a large proportion of CORE neurons responded to TUT plus other songs compared to SHELL neurons ( 0.43 versus 0.15) (Achiro and Bottjer, 2013). Approximately equal proportions of CORE and SHELL neurons showed responses only to OWN (CORE 0.15, SHELL 0.13). Thus SHELL neurons in anesthetized birds contain two distinct populations of neurons during early sensorimotor integration: one that responds only to the tutor song and a separate population that responds only to the bird's own song. In general, CORE neurons in anesthetized birds are much more broadly tuned than SHELL neurons and show little evidence of responsivity to tutor song (see below and Achiro \& Bottjer 2013). The current data did not replicate any of these patterns in sleeping birds (see Discussion and Table 3). 


\section{Response strengths were greater for songs that elicited a selective response}

Figure 4 (top) shows absolute values of response strengths within all CORE and SHELL neurons for responses to each song type (including both excitatory and suppressed responses). This measure revealed no difference in firing rates between songs in SHELL but a significant difference in CORE (Friedman test: CORE, $p=0.039 ;$ SHELL, $p=0.257$ ), reflecting a stronger response to JuvCon relative to other songs in CORE neurons. Given the relatively large proportion of significant responses to JuvCon song in both CORE and SHELL, we compared absolute values of response strengths across song types for the subset of neurons that responded significantly to JuvCon ( $n=19$ CORE, $n=21$ SHELL). Figure 4 (middle) shows that this subpopulation in both CORE and SHELL exhibited a significantly higher firing rate to JuvCon compared to the other three song types (Friedman tests, $p<0.0001$ in both CORE and SHELL; Wilcoxon signed-rank tests for JuvCon versus other song types always $p<0.004$ or lower, Benjamini-Hochberg corrected). To determine whether this selective increase in firing rate was restricted to JuvCon-responsive neurons, we calculated firing rates for each subset of neurons that showed a significant response to the remaining three song types. A similar pattern was obtained for OWN-responsive, AdlCon-responsive, and TUT-responsive neurons, showing that single neurons that responded significantly to a given song type also showed a higher firing rate to that song type compared to other song stimuli. For example, OWN-responsive neurons in both CORE and SHELL had significantly higher response strengths to OWN compared to all other songs (Fig. 4, bottom; Wilcoxon signed-rank tests for OWN versus other song types in SHELL always $p<0.001$; in CORE OWN versus AdICon $p=0.005$, versus JuvCon $p=0.003$; versus TUT $p=0.001$ ). (We did not perform statistical tests for TUT-responsive or AdICon-responsive neurons due to relatively low n's.)

Given the prevalence of suppressed responses to JuvCon songs (Tables 1, 2), we examined neural selectivity between pairs of stimuli for JuvCon-suppressed neurons by calculating the difference in response strength between song types (see Methods). Response strengths to OWN, AdICon, and TUT were subtracted from significantly suppressed JuvCon responses for each cell. A positive difference score indicates that a neuron preferred JuvCon song over comparison songs. Figure 5 shows cumulative distributions of difference scores in CORE versus SHELL neurons for JuvCon against each of the three other song types ( $n=13$ CORE, $n=19$ SHELL). CORE and SHELL neurons clearly showed the same degree of preference for JuvCon song (Kolmogorov-Smirnov tests, $p$ always $>0.87$ ). A similar pattern of selectivity in CORE versus SHELL neurons was obtained when we compared cumulative distributions of difference scores for OWN ( $n=9$ CORE, $n=18$ SHELL) against each of the three other song types (data not shown). Furthermore, very few neurons exhibited negative selectivity scores; the preponderance of positive scores in Figure 5 shows that cells that exhibited significant suppression to JuvCon almost never showed greater suppression to any other song stimulus. For example, only one SHELL neuron and no CORE neurons showed stronger suppression to AdlCon compared to JuvCon (Fig. 5). One-sample Wilcoxon signed-rank tests to assess whether the distributions were different from zero always yielded $p$ values of $<0.0001$. 


\section{Discussion}

$\underline{\text { Responses in awake versus sleeping or anesthetized states of adult animals }}$

Many studies have shown that neural responses to song playback in the motor pathway of the song system, including the cortical regions HVC and RA (Fig. 1), are greatly diminished or absent in awake adult male songbirds but can be unmasked under anesthesia or in sleep (Dave et al., 1998; Schmidt and Konishi, 1998; Dave and Margoliash, 2000; Cardin and Schmidt, 2003; Rauske et al., 2003; Cardin and Schmidt, 2004b, a). This pattern may reflect, at least in part, a general tendency for responses in auditory and/or sensorimotor brain regions to be suppressed during self-generated sounds (Suga and Shimozawa, 1974; Poulet and Hedwig, 2006, 2007; Eliades and Wang, 2008; Singla et al., 2017). For example, neurons in auditory cortex of marmosets show suppression during vocal production; however, responses to their self-generated vocalizations are unmasked when auditory feedback is altered by realtime frequency shifts delivered through headphones (Eliades \& Wang, 2008). One idea to arise from such findings is that learned signals from motor or other non-auditory inputs can predict auditory feedback and cancel responses to corresponding auditory sounds. A variant of this idea might explain the absence of song-evoked responses in awake songbirds; for example, motor circuits or pathways for efference copy might act to suppress auditory responses in an awake state even in the absence of active vocalizing. More broadly, the tendency for responses in awake or vocalizing animals to be suppressed is consistent with the idea that behavioral state can regulate a "gate" that controls auditory input.

In accord with this latter idea, the fact that responses to song playback in vocal-control regions of songbirds are absent in fully awake states but can be evoked under anesthesia or during sleep has been interpreted as changes in behavioral state, although no mechanism has been proposed as to the source of suppression in the awake state. In anesthetized and sleeping adult male songbirds, neurons throughout the song system are selectively tuned to each individual bird's own song relative to conspecific songs or their OWN songs played in mirror-image reverse (Margoliash, 1983, 1986; Margoliash and Fortune, 1992; Vicario and Yohay, 1993; Nick and Konishi, 2001; Cardin and Schmidt, 2003; Person and Perkel, 2007). Cardin \& Schmidt (2003) directly compared responses of HVC neurons in anesthetized, sleeping, and awake adult zebra finches; responses in both anesthetized and sleeping birds were consistently selective for OWN songs, whereas responses in waking birds were highly variable and not selective for OWN. Responses to playback in awake birds reflected the level of arousal: higher levels of arousal uniformly suppressed song-evoked responses in HVC (but had no effect in primary auditory cortex). The similarity of selective responses to OWN song in sleeping and anesthetized birds encouraged the idea that similar behavioral states underlie sleep and anesthesia.

\section{Responses in awake versus sleeping or anesthetized states of juvenile animals}

Very few studies have examined responses to song playback in juvenile songbirds during sleep. Using behavioral criteria to assess sleep during song development, Nick \& Konishi (2005b) reported that multiunit responses in HVC were strongest to tutor song in awake juvenile zebra finches during early sensorimotor integration, whereas OWN was preferred over tutor songs during sleep. Selectivity for OWN song changed over development in a pattern that tracked the current motor version of each bird's 
song (Nick and Konishi, 2005a). Spontaneous patterns of spiking in HVC during sleep also change over song development: both firing rate and bursting increase with age (Crandall Nick et al. 2007).

EEG patterns are not a reliable indicator of sleep in juvenile zebra finches; the amplitude of 1-4 $\mathrm{Hz}$ activity (delta, an indicator of slow-wave sleep) did not vary between sleep and wake states in zebra finches between 45-65 dph (Nick and Konishi, 2005b; Crandall et al., 2007). This finding is consistent with the fact that the cortical EEG does not show evidence of state-dependent activity in early postnatal mammals (Gramsbergen, 1976; Frank and Heller, 1997; Blumberg et al., 2005). Even after EEG patterns differentiate ( $\geq 12$ days postnatal in rodents), a long period of developmental changes ensues, which may be related to maturational changes that facilitate normal development of the nervous system (Khazipov and Luhmann, 2006; Cirelli and Tononi, 2015; Rensing et al., 2018).

Sleep is essential for vocal learning in juvenile zebra finches (Dave and Margoliash, 2000; Deregnaucourt et al., 2005; Crandall et al., 2007; Shank and Margoliash, 2009; Margoliash and Schmidt, 2010), which brings into question the influence of developmental changes during sleep in song-evoked activity, patterns of spontaneous spiking, and maturation of EEG patterns. Such changes within sensorimotor song regions may be related to substantial changes in the neural substrate for song learning (AlvarezBuylla et al., 1988; Nordeen and Nordeen, 1988a; Nordeen and Nordeen, 1988b; Herrmann and Arnold, 1991; Johnson and Bottjer, 1992; Nordeen et al., 1992; Johnson and Bottjer, 1993, 1994; Livingston and Mooney, 1997; Foster and Bottjer, 1998; lyengar et al., 1999; Kittelberger and Mooney, 1999; Livingston et al., 2000; Nixdorf-Bergweiler, 2001; lyengar and Bottjer, 2002a, b; Bottjer, 2005; Miller-Sims and Bottjer, 2012; Garst-Orozco et al., 2014; Chung and Bottjer, 2021). For example, axonal projections that are present only during early stages of sensorimotor integration may mediate temporally restricted processes of song learning (Miller-Sims and Bottjer, 2012; Chung and Bottjer, 2021); in addition, refinement of axonal connectivity may represent either a morphological correlate of song learning or a necessary prerequisite for acquisition of song (lyengar and Bottjer, 2002a, b). Developmental changes in sleep activity as well as in the neural substrate are likely to be related to changing patterns of responsivity to different song types at different stages of learning. A promising area for investigation lies in the extent to which developmental changes in sleep activity, the underlying neural substrate, selectivity for different song types, and maturation of vocal motor production are inter-related.

We are not aware of any previous studies that recorded the response of LMAN neurons to song playback during sleep in juvenile songbirds. Comparison of the present results in juvenile sleeping birds with those of Achiro and Bottjer (2013) in juvenile anesthetized birds clearly indicates that responses of LMAN neurons during sleep are substantially different from those recorded under urethane anesthesia in zebra finches during early sensorimotor integration. Salient differences in LMAN activity between this study and their previously published work are summarized in Table 3. Activity patterns in anesthetized birds revealed significant differences between CORE and SHELL for each of the measures listed in Table 3 , whereas none of these measures varied between regions in sleeping birds. Two particularly striking differences are the dominance of suppressed responses in the present study, and the lack of a prominent neuronal subpopulation that responds selectively to tutor song in SHELL as is seen in anesthetized birds. These differences raise the question of when and how the tutor-tuned SHELL 
neurons are utilized in the service of learning. Perhaps our sleep conditions were not conducive to eliciting responses from tutor-selective neurons, in which case they may have an important sleeprelated function under more normal conditions (e.g., in sleeping birds that are not wearing an electrode array attached to a cable). Or perhaps these neurons are actively involved in some aspect of learning during sleep but are gated off from activation via auditory playback. Another possibility is that tutortuned neurons can be activated during awake states (as for HVC neurons of juvenile birds, Nick \& Konishi, 2005), particularly during singing. If tutor-tuned neurons are activated only during singing in awake states, their activity might be difficult to identify in the context of motor-related activity (Achiro et al., 2017).

\section{Comparing urethane anesthesia and different sleep states}

Prior to beginning our study we assumed that responses to song playback during sleep in LMAN of juvenile birds would replicate results in anesthetized birds (which would have enabled direct comparison of playback-evoked patterns as in anesthetized birds and singing activity in awake birds). Because we did not intend to study sleep-related factors we made no effort to characterize different stages of sleep in relation to playback. Despite the fact that EEG patterns do not correlate with sleep stages in juvenile animals (Gramsbergen, 1976; Frank and Heller, 1997; Blumberg et al., 2005; Nick and Konishi, 2005b; Crandall et al., 2007; Cirelli and Tononi, 2015), different states of sleep and/or ultradian rhythms may nevertheless influence song responsivity. If so, different sleep states might provide a possible alternative explanation of the stark differences we observed between song-evoked activity in LMAN of sleeping versus anesthetized juvenile zebra finches. Robust responses to song playback are observed during slow wave sleep in HVC of adult zebra finches (Nick and Konishi, 2001). We are not aware of any studies that have compared song-evoked responses during REM (rapid eye movement) versus non-REM sleep. It would be interesting to correlate responsivity to song playback with EEG patterns in adult birds, taking into account that episodes of different sleep states are quite brief (less than $30 \mathrm{sec}$ in adult budgies) and slow wave sleep decreases through the night while REM sleep increases (Canavan and Margoliash, 2020). It is not clear how informative this approach might be in young songbirds given that EEG patterns are not a reliable indicator of sleep states in juvenile animals, although it is nevertheless possible that a given song type could elicit different neural responses in sensorimotor song regions depending on EEG activity.

The similarity of selective responses to OWN songs under sleep and anesthesia in HVC neurons of adult songbirds has encouraged the idea that behavioral states are highly similar between the two conditions. However, this idea has not been extensively tested in either birds or mammals. Some studies have suggested that urethane anesthesia mimics sleep, based on alternation of EEG patterns between a slowwave state that resembles non-REM sleep and an "activated" state with features of both REM sleep and waking (Clement et al., 2008; Pagliardini et al., 2013; Tisdale et al., 2018). Recent work has not supported this idea, based on detailed comparisons that measure several correlates to define conscious (waking) versus unconscious (sleeping) states, including power spectra of EEGs, synchronization between high-frequency (gamma) oscillations in different brain regions, directional patterns of 
activation, and temporal complexity of neural oscillations (Mashour and Hudetz, 2018; Kelz and Mashour, 2019; Mashour et al., 2020). A recent study that performed within-subject comparisons of sleep versus urethane anesthesia in rats reported that these EEG correlates of consciousness were significantly lower during anesthesia compared to sleep (Mondino et al., 2021). For example, normalized power of delta oscillations was higher during both "REM-like" and "non-REM-like" states of urethane anesthesia compared to their respective REM and non-REM states during sleep. In addition, despite qualitative similarities between REM and non-REM EEG patterns in sleep versus urethane anesthesia, clear-cut differences in temporal complexity existed between them. These authors concluded that urethane induces a pattern of "sustained unconsciousness" dissimilar from that of sleep. Thus, it seems likely that differences in patterns of brain activity between sleep and anesthesia could underlie the different responses to song playback that we observed in LMAN of juvenile zebra finches between the current study and previous work by Achiro and Bottjer (2013). If so, that would suggest that urethane anesthesia is more effective at removing one or more gates of song-evoked activity compared to sleep. 


\section{References}

Achiro JM, Bottjer SW (2013) Neural representation of a target auditory memory in a cortico-basal ganglia pathway. J Neurosci 33:14475-14488.

Achiro JM, Shen J, Bottjer SW (2017) Neural activity in cortico-basal ganglia circuits of juvenile songbirds encodes performance during goal-directed learning. Elife 6.

Adret P, Meliza CD, Margoliash D (2012) Song tutoring in presinging zebra finch juveniles biases a small population of higher-order song-selective neurons toward the tutor song. J Neurophysiol 108:1977-1987.

Alexander GE, Crutcher MD (1990) Functional architecture of basal ganglia circuits: neural substrates of parallel processing. Trends Neurosci 13:266-271.

Alvarez-Buylla A, Theelen M, Nottebohm F (1988) Birth of projection neurons in the higher vocal center of the canary forebrain before, during and after song learning. Proc Natl Acad Sci U S A 85:87228726.

Aronov D, Andalman AS, Fee MS (2008) A specialized forebrain circuit for vocal babbling in the juvenile songbird. Science 320:630-634.

Ashby FG, Turner BO, Horvitz JC (2010) Cortical and basal ganglia contributions to habit learning and automaticity. Trends Cogn Sci 14:208-215.

Benjamini Y, Hochberg Y (1995) Controlling the False Discovery Rate - a Practical and Powerful Approach to Multiple Testing. J Roy Stat Soc B Met 57:289-300.

Blumberg MS, Karlsson KA, Seelke AM, Mohns EJ (2005) The ontogeny of mammalian sleep: a response to Frank and Heller (2003). J Sleep Res 14:91-98.

Böhner J (1983) Song Learning in the Zebra Finch (Taeniopygia-Guttata) - Selectivity in the Choice of a Tutor and Accuracy of Song Copies. Anim Behav 31:231-237.

Böhner J (1990) Early acquisition of song in the zebra finch, Taeniopygia guttata. Anim Behav 39:369374.

Bottjer SW (2004) Developmental regulation of basal ganglia circuitry during the sensitive period for vocal learning in songbirds. Ann N Y Acad Sci 1016:395-415.

Bottjer SW (2005) Silent synapses in a thalamo-cortical circuit necessary for song learning in zebra finches. J Neurophysiol 94:3698-3707.

Bottjer SW, Altenau B (2010) Parallel pathways for vocal learning in basal ganglia of songbirds. Nat Neurosci 13:153-155.

Bottjer SW, Miesner EA, Arnold AP (1984) Forebrain lesions disrupt development but not maintenance of song in passerine birds. Science 224:901-903.

Brainard MS, Doupe AJ (2013) Translating birdsong: songbirds as a model for basic and applied medical research. Annu Rev Neurosci 36:489-517.

Canavan SV, Margoliash D (2020) Budgerigars have complex sleep structure similar to that of mammals. PLoS Biol 18:e3000929.

Cardin JA, Schmidt MF (2003) Song system auditory responses are stable and highly tuned during sedation, rapidly modulated and unselective during wakefulness, and suppressed by arousal. J Neurophysiol 90:2884-2899.

Cardin JA, Schmidt MF (2004a) Auditory responses in multiple sensorimotor song system nuclei are comodulated by behavioral state. J Neurophysiol 91:2148-2163.

Cardin JA, Schmidt MF (2004b) Noradrenergic inputs mediate state dependence of auditory responses in the avian song system. J Neurosci 24:7745-7753. 
Chung JH, Bottjer SW (2021) Developmentally regulated pathways for motor skill learning in songbirds. J Comp Neurol.

Cirelli C, Tononi G (2015) Cortical Development, Electroencephalogram Rhythms, and the Sleep/Wake Cycle. Biol Psychiat 77:1071-1078.

Clement EA, Richard A, Thwaites M, Ailon J, Peters S, Dickson CT (2008) Cyclic and sleep-like spontaneous alternations of brain state under urethane anaesthesia. PLoS One 3:e2004.

Cox J, Witten IB (2019) Striatal circuits for reward learning and decision-making. Nat Rev Neurosci.

Crandall SR, Adam M, Kinnischtzke AK, Nick TA (2007) HVC neural sleep activity increases with development and parallels nightly changes in song behavior. J Neurophysiol 98:232-240.

Dave AS, Margoliash D (2000) Song replay during sleep and computational rules for sensorimotor vocal learning. Science 290:812-816.

Dave AS, Yu AC, Margoliash D (1998) Behavioral state modulation of auditory activity in a vocal motor system. Science 282:2250-2254.

Deregnaucourt S, Mitra PP, Feher O, Pytte C, Tchernichovski O (2005) How sleep affects the developmental learning of bird song. Nature 433:710-716.

Doupe AJ (1997) Song- and order-selective neurons in the songbird anterior forebrain and their emergence during vocal development. J Neurosci 17:1147-1167.

Doupe AJ, Kuhl PK (1999) Birdsong and human speech: common themes and mechanisms. Annu Rev Neurosci 22:567-631.

Eliades SJ, Wang X (2008) Neural substrates of vocalization feedback monitoring in primate auditory cortex. Nature 453:1102-1106.

Elliott KC, Wu W, Bertram R, Johnson F (2014) Disconnection of a basal ganglia circuit in juvenile songbirds attenuates the spectral differentiation of song syllables. Dev Neurobiol 74:574-590.

Foster EF, Bottjer SW (1998) Axonal connections of the high vocal center and surrounding cortical regions in juvenile and adult male zebra finches. J Comp Neurol 397:118-138.

Frank MG, Heller HC (1997) Development of REM and slow wave sleep in the rat. Am J Physiol 272:R1792-1799.

Gale SD, Person AL, Perkel DJ (2008) A novel basal ganglia pathway forms a loop linking a vocal learning circuit with its dopaminergic input. J Comp Neurol 508:824-839.

Garst-Orozco J, Babadi B, Ölveczky BP (2014) A neural circuit mechanism for regulating vocal variability during song learning in zebra finches. Elife 3:e03697.

Gramsbergen A (1976) The development of the EEG in the rat. Dev Psychobiol 9:501-515.

Graybiel AM (2008) Habits, rituals, and the evaluative brain. Annu Rev Neurosci 31:359-387.

Gremel CM, Costa RM (2013) Orbitofrontal and striatal circuits dynamically encode the shift between goal-directed and habitual actions. Nature communications 4:2264.

Herrmann K, Arnold AP (1991) The development of afferent projections to the robust archistriatal nucleus in male zebra finches: a quantitative electron microscopic study. J Neurosci 11:20632074.

lyengar S, Bottjer SW (2002a) The role of auditory experience in the formation of neural circuits underlying vocal learning in zebra finches. J Neurosci 22:946-958.

Iyengar S, Bottjer SW (2002b) Development of individual axon arbors in a thalamocortical circuit necessary for song learning in zebra finches. J Neurosci 22:901-911.

lyengar S, Viswanathan SS, Bottjer SW (1999) Development of topography within song control circuitry of zebra finches during the sensitive period for song learning. J Neurosci 19:6037-6057.

Johnson F, Bottjer SW (1992) Growth and regression of thalamic efferents in the song-control system of male zebra finches. J Comp Neurol 326:442-450.

Johnson F, Bottjer SW (1993) Induced cell death in a thalamic nucleus during a restricted period of zebra finch vocal development. J Neurosci 13:2452-2462. 
Johnson F, Bottjer SW (1994) Afferent influences on cell death and birth during development of a cortical nucleus necessary for learned vocal behavior in zebra finches. Development 120:13-24.

Johnson F, Sablan MM, Bottjer SW (1995) Topographic organization of a forebrain pathway involved with vocal learning in zebra finches. J Comp Neurol 358:260-278.

Kelz MB, Mashour GA (2019) The Biology of General Anesthesia from Paramecium to Primate. Curr Biol 29:R1199-R1210.

Khazipov R, Luhmann HJ (2006) Early patterns of electrical activity in the developing cerebral cortex of humans and rodents. Trends in Neurosciences 29:414-418.

Kittelberger JM, Mooney R (1999) Lesions of an avian forebrain nucleus that disrupt song development alter synaptic connectivity and transmission in the vocal premotor pathway. J Neurosci 19:93859398.

Kojima S, Doupe AJ (2007) Song selectivity in the pallial-basal ganglia song circuit of zebra finches raised without tutor song exposure. J Neurophysiol 98:2099-2109.

Kojima S, Kao MH, Doupe AJ, Brainard MS (2018) The Avian Basal Ganglia Are a Source of Rapid Behavioral Variation That Enables Vocal Motor Exploration. J Neurosci 38:9635-9647.

Kupferschmidt DA, Juczewski K, Cui G, Johnson KA, Lovinger DM (2017) Parallel, but Dissociable, Processing in Discrete Corticostriatal Inputs Encodes Skill Learning. Neuron 96:476-489 e475.

Lewicki MS, Konishi M (1995) Mechanisms underlying the sensitivity of songbird forebrain neurons to temporal order. Proc Natl Acad Sci U S A 92:5582-5586.

Livingston FS, Mooney R (1997) Development of intrinsic and synaptic properties in a forebrain nucleus essential to avian song learning. J Neurosci 17:8997-9009.

Livingston FS, White SA, Mooney R (2000) Slow NMDA-EPSCs at synapses critical for song development are not required for song learning in zebra finches. Nat Neurosci 3:482-488.

Low PS, Shank SS, Sejnowski TJ, Margoliash D (2008) Mammalian-like features of sleep structure in zebra finches. Proc Natl Acad Sci U S A 105:9081-9086.

Ludwig KA, Miriani RM, Langhals NB, Joseph MD, Anderson DJ, Kipke DR (2009) Using a common average reference to improve cortical neuron recordings from microelectrode arrays. J Neurophysiol 101:1679-1689.

Luo M, Ding L, Perkel DJ (2001) An avian basal ganglia pathway essential for vocal learning forms a closed topographic loop. J Neurosci 21:6836-6845.

Mann NI, Slater PJB (1995) Song Tutor Choice by Zebra Finches in Aviaries. Anim Behav 49:811-820.

Mann NI, Slater PJB, Eales LA, Richards C (1991) The influence of visual stimuli on song tutor choice in the zebra finch, Taenopygia guttata Anim Behav 42:285-293.

Margoliash D (1983) Acoustic parameters underlying the responses of song-specific neurons in the white-crowned sparrow. J Neurosci 3:1039-1057.

Margoliash D (1986) Preference for autogenous song by auditory neurons in a song system nucleus of the white-crowned sparrow. J Neurosci 6:1643-1661.

Margoliash D, Konishi M (1985) Auditory representation of autogenous song in the song system of white-crowned sparrows. Proc Natl Acad Sci U S A 82:5997-6000.

Margoliash D, Fortune ES (1992) Temporal and harmonic combination-sensitive neurons in the zebra finch's HVc. J Neurosci 12:4309-4326.

Margoliash D, Schmidt MF (2010) Sleep, off-line processing, and vocal learning. Brain Lang 115:45-58.

Mashour GA, Hudetz AG (2018) Neural Correlates of Unconsciousness in Large-Scale Brain Networks. Trends Neurosci 41:150-160.

Mashour GA, Roelfsema P, Changeux JP, Dehaene S (2020) Conscious Processing and the Global Neuronal Workspace Hypothesis. Neuron 105:776-798. 
Miller-Sims VC, Bottjer SW (2012) Auditory experience refines cortico-basal ganglia inputs to motor cortex via remapping of single axons during vocal learning in zebra finches. J Neurophysiol 107:1142-1156.

Mondino A, González J, Li D, Mateos D, Osorio L, Cavelli M, Costa A, Vanini G, Mashour G, Torterolo P (2021) Urethane Anesthesia Exhibits Neurophysiological Correlates of Unconsciousness and is Distinct from Sleep. bioRxiv. doi: https://doi.org/10.1101/2021.09.21.461281

Nick TA, Konishi M (2001) Dynamic control of auditory activity during sleep: correlation between song response and EEG. Proc Natl Acad Sci U S A 98:14012-14016.

Nick TA, Konishi M (2005a) Neural auditory selectivity develops in parallel with song. J Neurobiol 62:469481.

Nick TA, Konishi M (2005b) Neural song preference during vocal learning in the zebra finch depends on age and state. J Neurobiol 62:231-242.

Nixdorf-Bergweiler BE (2001) Lateral magnocellular nucleus of the anterior neostriatum (LMAN) in the zebra finch: neuronal connectivity and the emergence of sex differences in cell morphology. Microsc Res Tech 54:335-353.

Nordeen EJ, Nordeen KW (1988a) Sex and regional differences in the incorporation of neurons born during song learning in zebra finches. J Neurosci 8:2869-2874.

Nordeen EJ, Grace A, Burek MJ, Nordeen KW (1992) Sex-dependent loss of projection neurons involved in avian song learning. J Neurobiol 23:671-679.

Nordeen KW, Nordeen EJ (1988b) Projection neurons within a vocal motor pathway are born during song learning in zebra finches. Nature 334:149-151.

Pagliardini S, Gosgnach S, Dickson CT (2013) Spontaneous sleep-like brain state alternations and breathing characteristics in urethane anesthetized mice. PLoS One 8:e70411.

Paterson AK, Bottjer SW (2017) Cortical inter-hemispheric circuits for multimodal vocal learning in songbirds. J Comp Neurol 525:3312-3340.

Person AL, Perkel DJ (2007) Pallidal neuron activity increases during sensory relay through thalamus in a songbird circuit essential for learning. J Neurosci 27:8687-8698.

Person AL, Gale SD, Farries MA, Perkel DJ (2008) Organization of the songbird basal ganglia, including area X. J Comp Neurol 508:840-866.

Poulet JF, Hedwig B (2006) The cellular basis of a corollary discharge. Science 311:518-522.

Poulet JF, Hedwig B (2007) New insights into corollary discharges mediated by identified neural pathways. Trends Neurosci 30:14-21.

Rauske PL, Shea SD, Margoliash D (2003) State and neuronal class-dependent reconfiguration in the avian song system. J Neurophysiol 89:1688-1701.

Redgrave $\mathrm{P}$, Rodriguez M, Smith Y, Rodriguez-Oroz MC, Lehericy S, Bergman H, Agid Y, DeLong MR, Obeso JA (2010) Goal-directed and habitual control in the basal ganglia: implications for Parkinson's disease. Nat Rev Neurosci 11:760-772.

Rensing N, Moy B, Friedman JL, Galindo R, Wong M (2018) Longitudinal analysis of developmental changes in electroencephalography patterns and sleep-wake states of the neonatal mouse. PLoS One 13:e0207031.

Roper A, Zann R (2006) The onset of song learning and song tutor selection in fledgling zebra finches. Ethology 112:458-470.

Scharff C, Nottebohm F (1991) A comparative study of the behavioral deficits following lesions of various parts of the zebra finch song system: implications for vocal learning. J Neurosci 11:2896-2913.

Schmidt MF, Konishi M (1998) Gating of auditory responses in the vocal control system of awake songbirds. Nat Neurosci 1:513-518.

Shank SS, Margoliash D (2009) Sleep and sensorimotor integration during early vocal learning in a songbird. Nature 458:73-77. 
Singla S, Dempsey C, Warren R, Enikolopov AG, Sawtell NB (2017) A cerebellum-like circuit in the auditory system cancels responses to self-generated sounds. Nat Neurosci 20:943-950.

Solis MM, Doupe AJ (1997) Anterior forebrain neurons develop selectivity by an intermediate stage of birdsong learning. J Neurosci 17:6447-6462.

Solis MM, Doupe AJ (1999) Contributions of tutor and bird's own song experience to neural selectivity in the songbird anterior forebrain. J Neurosci 19:4559-4584.

Solis MM, Doupe AJ (2000) Compromised neural selectivity for song in birds with impaired sensorimotor learning. Neuron 25:109-121.

Suga N, Shimozawa T (1974) Site of neural attenuation of responses to self-vocalized sounds in echolocating bats. Science 183:1211-1213.

Szymczak JT, Kaiser W, Helb HW, Beszczynska B (1996) A study of sleep in the European blackbird. Physiol Behav 60:1115-1120.

Thorn CA, Atallah H, Howe M, Graybiel AM (2010) Differential dynamics of activity changes in dorsolateral and dorsomedial striatal loops during learning. Neuron 66:781-795.

Tisdale RK, Tieri L, Rattenborg NC, Beckers GJL, Lesku JA (2018) Spectral Properties of Brain Activity Under Two Anesthetics and Their Potential for Inducing Natural Sleep in Birds. Front Neurosci 12:881.

Turner RS, Desmurget M (2010) Basal ganglia contributions to motor control: a vigorous tutor. Curr Opin Neurobiol 20:704-716.

Vicario DS, Yohay KH (1993) Song-selective auditory input to a forebrain vocal control nucleus in the zebra finch. J Neurobiol 24:488-505.

Volman SF (1993) Development of neural selectivity for birdsong during vocal learning. J Neurosci 13:4737-4747.

Yin $\mathrm{HH}$, Knowlton BJ (2006) The role of the basal ganglia in habit formation. Nat Rev Neurosci 7:464-476.

Yin HH, Mulcare SP, Hilario MR, Clouse E, Holloway T, Davis MI, Hansson AC, Lovinger DM, Costa RM (2009) Dynamic reorganization of striatal circuits during the acquisition and consolidation of a skill. Nat Neurosci 12:333-341.

Zevin JD, Seidenberg MS, Bottjer SW (2004) Limits on reacquisition of song in adult zebra finches exposed to white noise. J Neurosci 24:5849-5862. 


\section{Figure Legends}

Figure 1. A simplified schematic of cortico-basal ganglia circuits that mediate vocal learning and behavior. The cortical nucleus LMAN comprises CORE (gray) and SHELL (red) subregions which form parallel recurrent loops through the basal ganglia and dorsal thalamus. LMAN-SHELL also forms a transcortical loop via Ald that converges with basal ganglia loops in the same dorsal thalamic zone. A transient projection from LMAN-CORE to Ald is present only in juvenile birds and creates a site of integration between CORE and SHELL pathways in Ald during early sensorimotor learning (denoted by dotted line). The dorsal thalamic zone feeds back to LMAN and feeds forward to HVC via medial MAN (latter pathway not shown for clarity). A specific region of the basal ganglia known as Area $\mathrm{X}$ is dedicated to functions for vocal learning and includes both striatal and pallidal cells. Abbreviations: RA: robust nucleus of the arcopallium; Ald: dorsal intermediate arcopallium; HVC: high vocal center; LMAN: lateral magnocellular nucleus of the anterior nidopallium.

Figure 2. Proportion of significant responses to each song stimulus in CORE (gray) versus SHELL (red) neurons. Top: Proportions of excited and suppressed responses to playback of each song type (see Table 1). ${ }^{*} p=0.04, * * p=0.01, * * * p=0.003$. Bottom: Proportions of suppressed responses to each song type. ${ }^{*} p=0.03, * * p=0.01, * * * p=0.006$. AdICon, Adult Conspecific song; JuvCon, Juvenile Conspecific song; TUT, tutor song; OWN, bird's own song. $n=44$ responses in 35 CORE neurons; $n=63$ responses in 49 SHELL neurons.

Figure 3. Single neurons were selectively tuned in both CORE and SHELL. Left: proportions of neurons that responded to different numbers of stimuli out of the four songs played; most neurons ( $75 \%$ ) responded to only one song stimulus in both CORRE and SHELL. Right: charts in which each row indicates the song stimuli to which each neuron responded $(n=44$ responses in 35 CORE neurons; $n=63$ responses in 49 SHELL neurons).

Figure 4. Standardized response strengths to each song stimulus across neurons. Box-and-whisker plots depict medians and first and third quartiles, whiskers indicate minimum and maximum values, and circles represent individual data points; all graphs depict absolute values (ABS) of standardized response strengths within CORE (gray) and SHELL (red) neurons. Top: response strengths to each song type (including both excitatory and suppressed responses, both significant and non-significant) for all CORE and SHELL neurons ( $n=35$ CORE, $n=49$ SHELL). Middle: response strengths for the subset of CORE and SHELL neurons that showed a significant response to JuvCon song ( $n=19$ CORE, $n=21$ SHELL). Bottom: response strengths for the subset of CORE and SHELL neurons that showed a significant response to OWN song ( $n=11$ CORE, $n=21$ SHELL).

Figure 5. CORE and SHELL neurons were equally selective for JuvCon song. Each panel shows cumulative distribution functions of selectivity scores for JuvCon song compared to AdICon (top), TUT, (middle) and OWN (bottom) ( $n=13$ CORE, $n=19$ SHELL). Positive difference scores indicate a preference for JuvCon song over comparison songs, and show that both CORE (gray) and SHELL (red) neurons preferred JuvCon song over comparison songs to the same extent. 


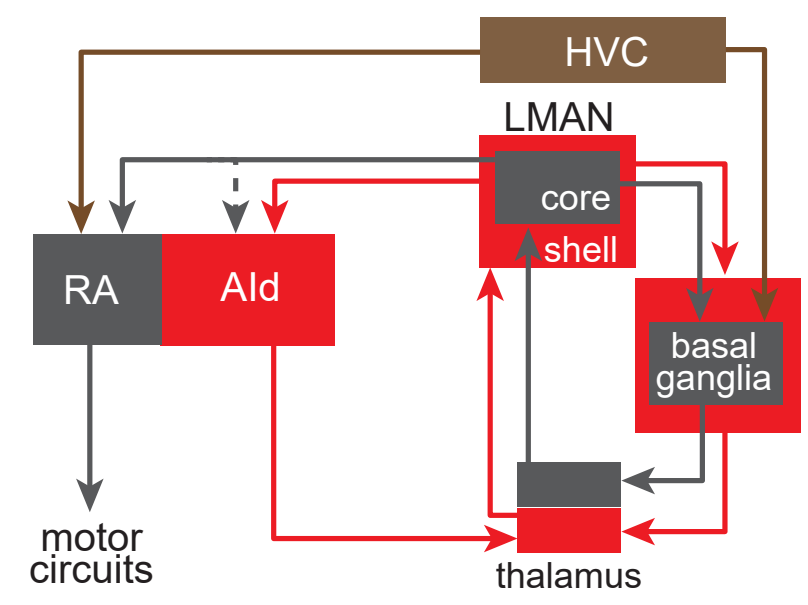

Figure 1. A simplified schematic of cortico-basal ganglia circuits that mediate vocal learning and behavior. The cortical nucleus LMAN comprises CORE (gray) and SHELL (red) subregions which form parallel recurrent loops through the basal ganglia and dorsal thalamus. LMAN-SHELL also forms a trans-cortical loop via Ald that converges with basal ganglia loops in the same dorsal thalamic zone. A transient projection from LMAN-CORE to Ald is present only in juvenile birds and creates a site of integration between CORE and SHELL pathways in Ald during early sensorimotor learning (denoted by dotted line). The dorsal thalamic zone feeds back to LMAN and feeds forward to HVC via medial MAN (latter pathway not shown for clarity). A specific region of the basal ganglia known as Area $X$ is dedicated to functions for vocal learning and includes both striatal and pallidal cells. Abbreviations: RA: robust nucleus of the arcopallium; Ald: dorsal intermediate arcopallium; HVC: high vocal center; LMAN: lateral magnocellular nucleus of the anterior nidopallium. 


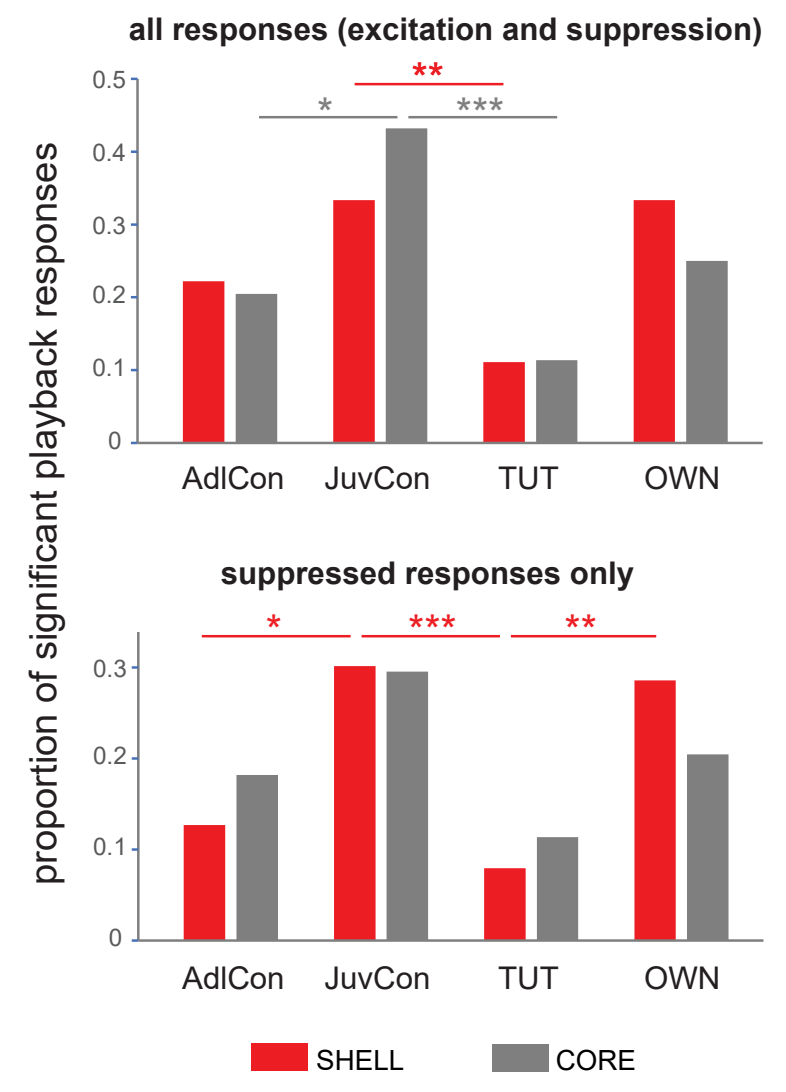

Figure 2. Proportion of significant responses to each song stimulus in CORE (gray) versus SHELL (red) neurons. Top: Proportions of excited and suppressed responses to playback of each song type (see Table 1). ${ }^{*} p=0.04,{ }^{* *} p=0.01,{ }^{* * *} p=0.003$. Bottom: Proportions of suppressed responses to each song type. ${ }^{*} p=0.03,{ }^{* *} p=0.01,{ }^{* * *} p=0.006$. AdICon, Adult Conspecific song; JuvCon, Juvenile Conspecific song; TUT, tutor song; OWN, bird's own song. $\mathrm{n}=44$ responses in 35 CORE neurons; $\mathrm{n}=$ 63 responses in 49 SHELL neurons. 


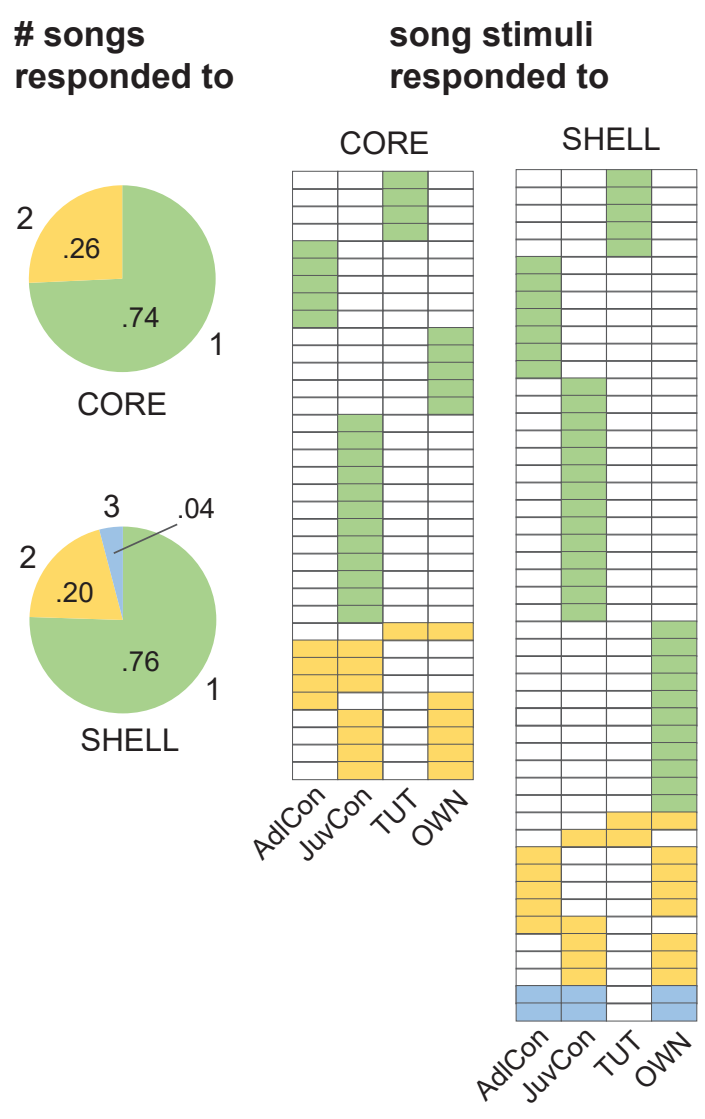

Figure 3. Single neurons were selectively tuned in both CORE and SHELL. Left: proportions of neurons that responded to different numbers of stimuli out of the four songs played; most neurons ( 75\%) responded to only one song stimulus in both CORRE and SHELL. Right: charts in which each row indicates the song stimuli to which each neuron responded $(n=44$ responses in 35 CORE neurons; $n=63$ responses in 49 SHELL neurons). 

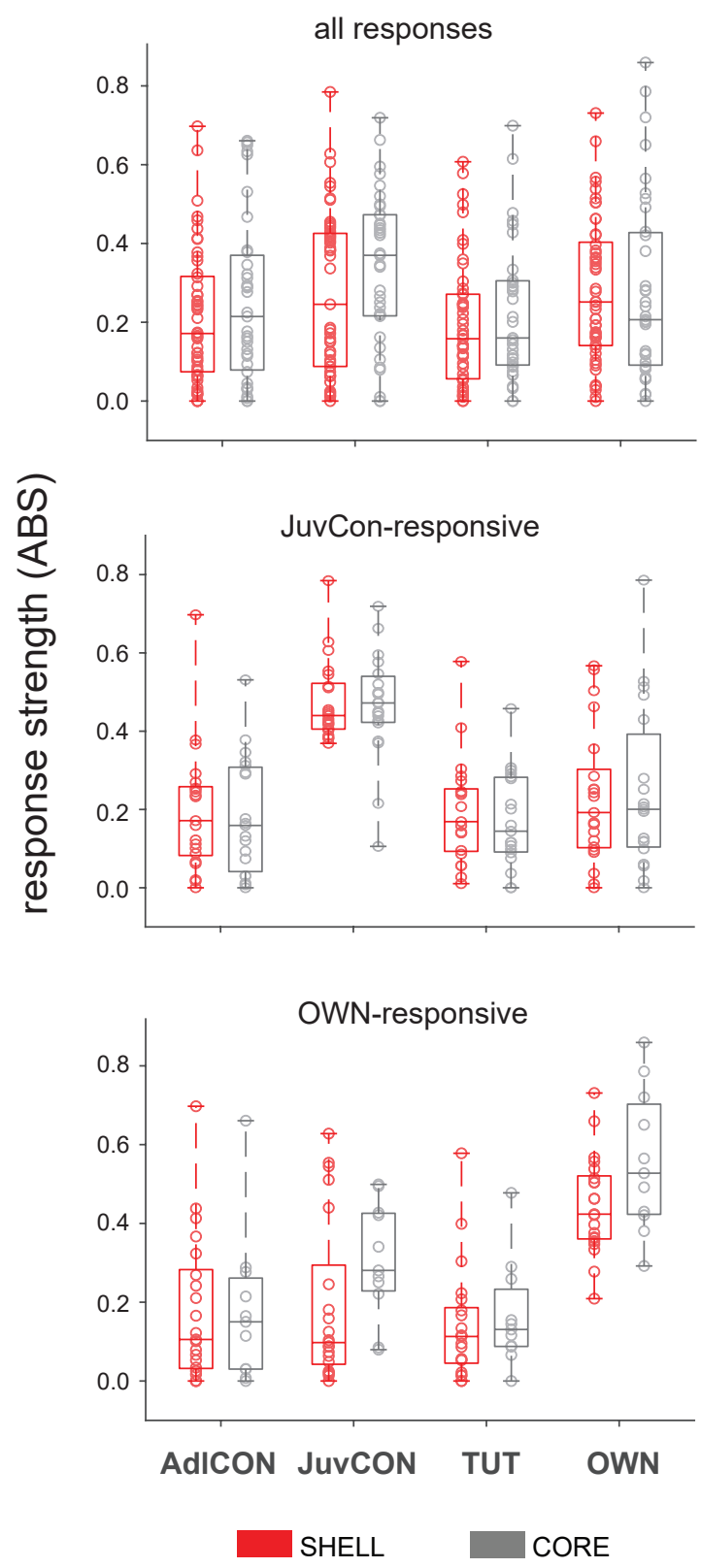

Figure 4. Standardized response strengths to each song stimulus across neurons. Box-andwhisker plots depict medians and first and third quartiles, whiskers indicate minimum and maximum values, and circles represent individual data points; all graphs depict absolute values (ABS) of standardized response strengths within CORE (gray) and SHELL (red) neurons. Top: response strengths to each song type (including both excitatory and suppressed responses, both significant and non-significant) for all CORE and SHELL neurons ( $n=35$ CORE, $n=49$ SHELL). Middle: response strengths for the subset of CORE and SHELL neurons that showed a significant response to JuvCon song ( $n=19$ CORE, $n=21$ SHELL). Bottom: response strengths for the subset of CORE and SHELL neurons that showed a significant response to OWN song ( $n=11$ CORE, $\mathrm{n}=21$ SHELL). 

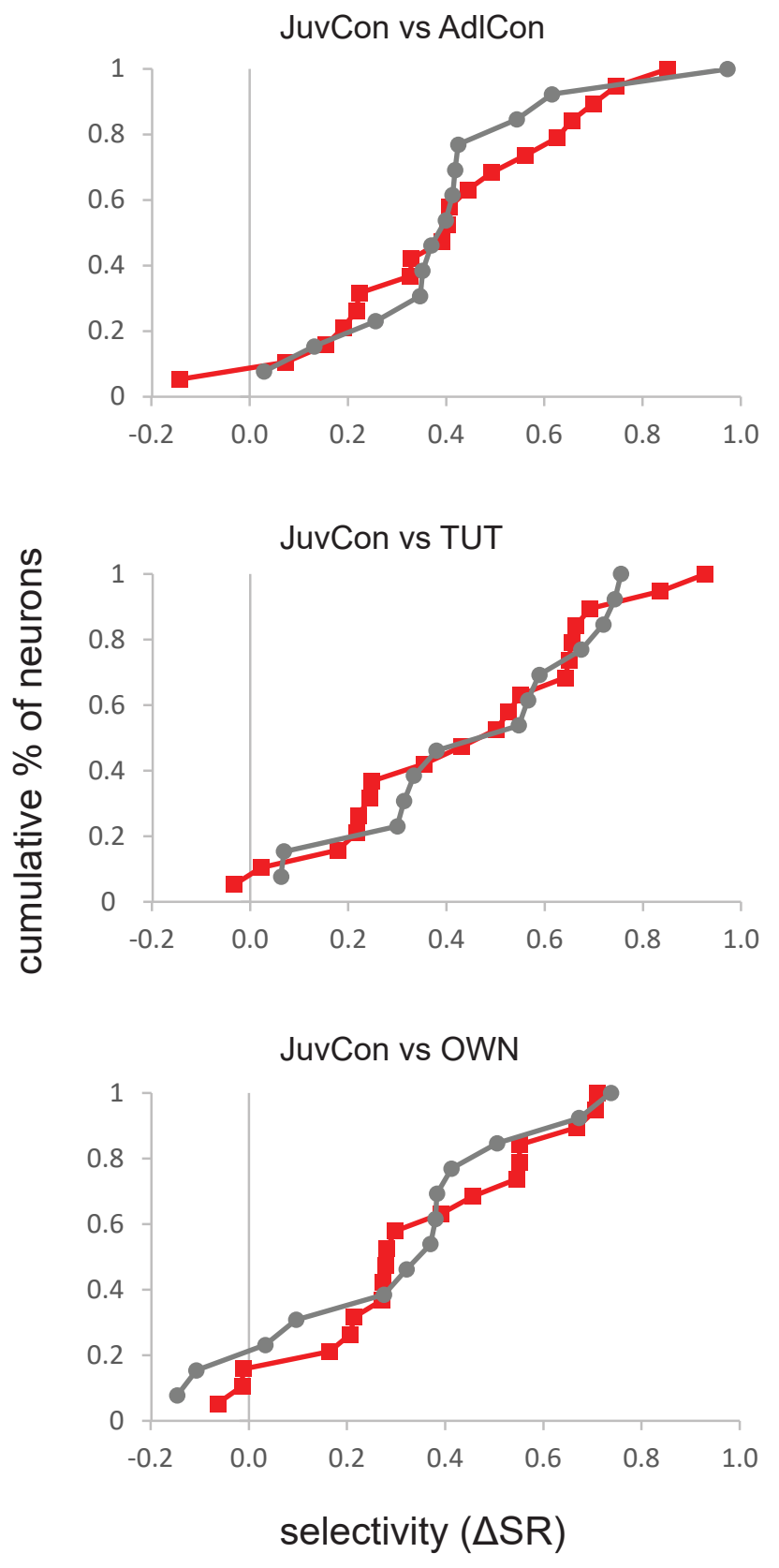

SHELL

CORE

Figure 5. CORE and SHELL neurons were equally selective for JuvCon song. Each panel shows cumulative distribution functions of selectivity scores for JuvCon song compared to AdICon (top), TUT, (middle) and OWN (bottom) ( $n=13$ CORE, $n=19$ SHELL). Positive difference scores indicate a preference for JuvCon song over comparison songs, and show that both CORE (gray) and SHELL (red) neurons preferred JuvCon song over comparison songs to the same extent. 
TABLE 1 Proportions of significant responses by song type

$\begin{array}{lllll}\text { SHELL } & \text { AdICon } & \text { JuvCon } & \text { TUT } & \text { OWN } \\ \text { all responses } & 0.22(14 / 63) & 0.33(21 / 63) & 0.11(7 / 63) & 0.33(21 / 63) \\ \text { excited } & 0.10(6 / 63) & 0.03(2 / 63) & 0.03(2 / 63) & 0.05(3 / 63) \\ \text { suppressed } & 0.13(8 / 63) & 0.30(19 / 63) & 0.08(5 / 63) & 0.29(18 / 63) \\ & & & & \\ \text { CORE } & & & & \\ \text { all responses } & 0.20(9 / 44) & 0.43(19 / 44) & 0.11(5 / 44) & 0.25(11 / 44) \\ \text { excited } & 0.02(1 / 44) & 0.14(6 / 44) & 0.0(0 / 44) & 0.05(2 / 44) \\ \text { suppressed } & 0.18(8 / 44) & 0.30(13 / 44) & 0.11(5 / 44) & 0.21(9 / 44)\end{array}$

TABLE 2 Proportions of neurons by response type

SHELL

$(n=49)$

excitation only

suppression only

both

\begin{tabular}{|r|r|} 
\# cells & proportion \\
\hline 8 & 0.163 \\
\hline 37 & 0.755 \\
\hline 4 & 0.082 \\
\hline
\end{tabular}

CORE

$(\mathrm{n}=35) \quad$ \# cells proportion

\begin{tabular}{|r|r|}
\hline 4 & 0.114 \\
\hline 26 & 0.743 \\
\hline 5 & 0.143 \\
\hline
\end{tabular}

TABLE 3 Comparison of current results with those of Achiro \& Bottjer 2013

$\%$ song-evoked neurons ${ }^{\mathrm{a}}$

$\%$ suppressed neurons ${ }^{b}$

\% TUT-only responsive neurons ${ }^{c}$

selectivity score JuvCon versus TUT ${ }^{d}$

selectivity score JuvCon versus $\mathrm{OWN}^{\mathrm{d}}$

\begin{tabular}{|c|c|c|c|c|}
\hline \multicolumn{2}{|c|}{ Anesthetized } & & \multicolumn{2}{c|}{ Sleeping } \\
\hline CORE & SHELL & & CORE & SHELL \\
\hline 89 & 68 & & 53 & 47 \\
\hline 0 & $\sim 20$ & & 74 & 76 \\
\hline 4 & 28 & & 11 & 10 \\
\hline 0.13 & 0.43 & & 0.47 & 0.45 \\
\hline 0.18 & 0.39 & & 0.30 & 0.34 \\
\hline
\end{tabular}

Anesthetized values are taken from Achiro \& Bottjer (2013); sleeping values are taken from current study. The mean age of birds at which recordings were made in Achiro \& Bottjer (2013) was $45.5 \mathrm{dph}$ (range 43-47); the mean age of birds from recordings in the current study was $48.5 \mathrm{dph}$ (range 43 to 53). a percentage of neurons that responded to playback of at least one song.

${ }^{b}$ percentage of neurons that were suppressed by song playback (cells that showed suppression only are included for both studies).

c percentage of neurons that gave a significant response only to TUT and not to any other stimulus (out of 5 songs for Achiro \& Bottjer, out of 4 songs for the current study). 
Table 3 (continued)

${ }^{d}$ Selectivity scores for JuvCon versus TUT, OWN refer to average difference scores between standardized response strengths (see Methods). Scores from present study are for suppressed responses among JuvCon-selective cells, while scores from Achiro \& Bottjer are for cells for TUTselective and OWN- selective cells, respectively (Achiro \& Bottjer scores include excited responses for CORE and excited and suppressed responses for SHELL); we chose to present scores for JuvConresponsive cells from this study since so few neurons responded to TUT (Table 1 ). 